\title{
Electroencephalogram Based Reaction Time Prediction With Differential Phase Synchrony Representations Using Co-Operative Multi-Task Deep Neural Networks
}

\author{
Tharun Kumar Reddy, Vipul Arora ${ }^{\circledR}$, Satyam Kumar, Laxmidhar Behera ${ }^{\circledR}$, Senior Member, IEEE, \\ Yu-Kai Wang, Member, IEEE, and Chin-Teng Lin, Fellow, IEEE
}

\begin{abstract}
Driver drowsiness is receiving a lot of deliberation as it is a major cause of traffic accidents. This paper proposes a method which utilizes the fuzzy common spatial pattern optimized differential phase synchrony representations to inspect electroencephalogram (EEG) synchronization changes from the alert state to the drowsy state. EEG-based reaction time prediction and drowsiness detection are formulated as primary and ancillary problems in the context of multi-task learning. Statistical analysis results suggest that our method can be used to distinguish between alert and drowsy state of mind. The proposed Multi-Task DeepNet (MTDNN) performs superior to the baseline regression schemes, like support vector regression (SVR), least absolute shrinkage and selection operator, ridge regression, K-nearest neighbors, and adaptive neuro fuzzy inference scheme (ANFIS), in terms of root mean squared error (RMSE), mean absolute percentage error (MAPE), and correlation coefficient (CC) metrics. In particular, the best performing multi-task network MTDNN $_{5}$ recorded a $15.49 \%$ smaller RMSE, a $27.15 \%$ smaller MAPE, and a $10.13 \%$ larger CC value than SVR.
\end{abstract}

Index Terms-Brain Computer Interface (BCI), Deep Neural Network (DNN), One Versus Rest (OVR), Reaction Time (RT), Multi-Task Learning, Root Mean Squared Error (RMSE), Mean Absolute Percentage Error (MAPE) and Correlation Coefficient (CC).

Manuscript received July 15, 2018; revised September 3, 2018 and September 24, 2018; accepted October 5, 2018. This work was supported in part by the Australian Research Council (ARC) under discovery Grants DP180100670 and DP180100656, in part by the Ministry of Human Resource Development (MHRD), Government of India, under Project MHRD-EE-2016150, and in part by the Army Research Laboratory and was accomplished under Cooperative Agreement Numbers W911NF-10-2-0022 and W911NF-10-D-0002/TO 0023. The work of T. K. Reddy was supported under Project TCS/CS/2011191A. (Corresponding author: Laxmidhar Behera.)

T. K. Reddy, V. Arora, S. Kumar, and L. Behera are with the Department of Electrical Engineering, Indian Institute of Technology Kanpur, Kanpur 208016, India (e-mail: tharun@iitk.ac.in; vipular.iitk@gmail.com; satyamk@iitk.ac.in; lbehera@iitk.ac.in).

Y.-K. Wang and C.-T. Lin are with the Centre for Artificial Intelligence, Faculty of Engineering and Information Technology, University of Technology Sydney, Ultimo, NSW 2007, Australia (e-mail: yukai.wang@uts.edu.au; chintenglin@gmail.com)

This paper has supplementary downloadable material available at http://ieeexplore.ieee.org, provided by the authors. This includes two PDFs that show a more detailed performance analysis of results and implementation details comparing performance of the features and a computational complexity analysis of the proposed approach. This material is $467 \mathrm{~KB}$ in size.

Digital Object Identifier 10.1109/TETCI.2018.2881229

\section{INTRODUCTION}

D ROWSINESS (fatigue), also mentioned as sleepiness, signifies "the tendency to fall asleep". A major shift leading to the deployment of drowsiness detection systems in vehicles is largely attributed to the WHO report in 2013, which stated that almost almost $6 \%$ of world's road accidents are caused by drivers in drowsy state [1]. Some recent developments in drowsiness detection research are discussed below.

Among various physiological signals, EEG is one of the most reliable indicators because it is in close conjunction with mental and physical activities [2]. Several methods proposed in literature for detecting fatigue by EEG can be categorized into amplitude and phase based approaches. At first, we summarize the amplitude based approaches. Budi et al. [3] used EEG spectral feature segments to analyze four algorithms for detecting fatigue. They demonstrated that the ratio of the total spectra power in theta and alpha bands to the power in beta band witnessed a greater upsurge over time with drowsiness phenomenon. Wei et al. [4] defined a term, the Level of Session Generalizability (LSG) through a novel Transfer Learning (TL) based method. Their method utilizes a subjects pilot data to select ancillary data from other subjects to enhance the performance of an EEG based BCI for drowsiness detection. Authors in [5] propose regression with Random Forest on multiband power features providing a highly accurate fatigue index using only three electrode positions. [6] contains two novel driving fatigue prediction metrics. First integrated fatigue metric is based on power spectrum density analysis with subject specific channel selection and second metric is based on sample entropy analysis from ' $\mathrm{O} 1 \mathrm{~h}$ ' and 'O2h' electrodes. A. Saha et al. [7] used motor planning phase to detect cognitive failure in driving using type-2 fuzzy classifiers.

The phase based approaches for detecting drowsiness are discussed next. Phase based analysis has also been demonstrated to be the cynosure of functional neural connectivity inference [8]. Phase Synchronization (PS) or phase based analysis can detect the spatial lateralization of drowsiness phenomenon. This approach studies the interplay between signal pairs through the functional relationships of instantaneous phase among the signals independent of their amplitudes. Thus, the notion of PS [9] 
is fundamental for neuronal information processing within the brain region as well as communication between different brain regions. Lachaux et al. [10] proposed Phase Locking Value (PLV) statistic to quantify the frequency specific synchronization between the neural signals. However, since PLV is a temporal measure of synchrony across the trials, it is not suitable for trial wise analysis. To measure trial wise phase synchrony information, a new statistical single trial PLV was formulated [11]. Caramia et al. [12] proposed a modified single trial PLV but it did not account for lead lag behavior between EEG signals. Kumar et al. [13] proposed to extract features based on Instantaneous Phase Difference (IPD) for trial wise analysis. Further, [14] explored Mean Phase Coherence (MPC) with large and local scale synchrony for fatigue detection. In this work, authors adapt the DPS representations for regression which are an outcome of integration of IPD with fuzzy CSPR-OVR [15] framework. Authors further validate the proposed DPS features on the reaction time dataset from an EEG based lane-keeping task.

Thus, a large amount of literature already deals with signal processing for BCIs based on EEG classification problems, but research on EEG regression problems is mostly neglected till now. Some of the prominent EEG based regression problems are: estimation of continuous workload levels [16], Reaction Time (RT) for the EEG-tracked SVIPT [17] and RT prediction for the EEG based PVT and lane-keeping tasks [18].

After the EEG signal is obtained, the regression process involves several steps: 1) Signal pre-processing to enhance the Signal to noise ratio (SNR). Filters in frequency realm such as low-pass filters, band-pass filters, band-stop filters, and spatial filters such as independent component analysis (ICA), SPoC [19], CSP and fuzzy CSSP [20] are frequently used here. 2) Feature representations to shape relevant predictors, e.g., Riemannian Geometry features [18] and EEG power band features [15]. 3) Regressors to estimate the continuous valued variable, e.g., Ordinary Linear Regression (OLR) [21], Ridge Regression [22], LASSO [15], [18], K-nearest neighbors (kNN) [15], fuzzy neural networks [23], Transfer Learning [4], [24], [25], active learning [26], domain adaptation [27], multiview learning [28], multitask learning [29] etc.

In the multitask learning setup, there are multiple tasks, each of which is a general task such as supervised task, unsupervised task, semi-supervised task etc. A handful of these tasks, or a portion of them are related to each other. Cooperative training among these tasks can lead to a greater performance enhancement compared to training the tasks one at a time [30]. In BCIs, a task is usually considered a unique recording session, either for an individual or multiple subjects [31]. In [32], authors demonstrated the effectiveness of multitask learning for classification of motor imagery trials. The multitask learning approach proved to be robust against misclassification in different experimental conditions. Furthermore, multitask learning technique efficiently estimates spatial filters for classification of motor imagery in subjects with no prior training data.

RT denotes the time period between the onset of the lane deviation and the onset of the response and is used as an objective measure of the drowsiness (DS) level during each lane departure event [33]. Since EEG drowsiness estimation and EEG reaction time prediction are two related problems, where approximate solution to either of them helps to solve the other, Hence, multitask learning can be applied in this scenario.

The primary objectives of this study are:

- To demonstrate the utility of phase based feature representations for EEG based drowsiness detection.

- To model the drivers' drowsiness detection as a multi-task learning problem based on DPS-FCSPR-OVR feature representations and train intelligent models for the proposed tasks.

The major contributions of this work are:

- Novel DPS-FCSPR-OVR representations are proposed to demonstrate the utility of phase based EEG representations for EEG based RT prediction.

- A novel MTDNN framework with a supervised pretraining and fine tuning steps is proposed.

- Extensive experiments (including comparison with advanced regression models) are carried out to confirm the effectiveness of the proposed method in EEG based RT prediction.

The novelty of the proposed method is highlighted as follows:

- The utility of phase based feature representations is very scarcely studied in literature for EEG based drowsiness detection problem. For addressing this issue, the DPSFCSPR-OVR representations are adopted to train intelligent models.

- In the multitask BCI literature, the concept of task is usually limited to either a subject, session etc. For the first time, we extend the notion of BCI task to address two BCI problems: drowsiness detection and RT prediction.

This paper is organized as follows: Section II presents the formulation of proposed DPS-FCSPR-OVR representations. Section III evaluates their performance on the EEG lane keeping task. Section IV leads us to the proposed co-operative DNN based multitask approach (MTDNN). Section V evaluates the proposed MTDNN approach with several baseline DeepNets and other regression schemes. Finally, discussion and concluding remarks are provided in Section VI and VII respectively.

\section{DifFERENTIAL PHASE SYNCHRONY (DPS) REPRESENTATIONS}

\section{A. Fuzzy CSPR-OVR}

Let $\mathbf{X}_{n} \in \mathbb{R}^{C \times T} n \in\{1,2, \ldots, N\}$ denote the $n$th EEG trial, where $C$ denotes number of channels and $T$ denotes number of time samples per trial. Trial $\mathbf{X}_{n}$ constitutes a band pass filtered signal whose mean is removed from each of the channels. Using the concept of fuzzy sets, we define fuzzy classes (assume $M$ fuzzy classes) to generalize to regression problems. Next, the interval range $[0,100]$ is used to generate $M+1$ regions and let us denote the boundary partition points by $\left\{y_{p_{m}}\right\}, m=$ $\{1, \ldots, M\}$. To mention

$$
P_{m}=\frac{100 \cdot m}{M+1}, \quad m=1, \ldots, M
$$

Each $y_{p_{m}}$ is the $P_{m}$ percentile value of the training set of RTs. Next, we define $M$ fuzzy classes and categorize training RT values into one of the $M$ fuzzy classes, in a manner analogous to 


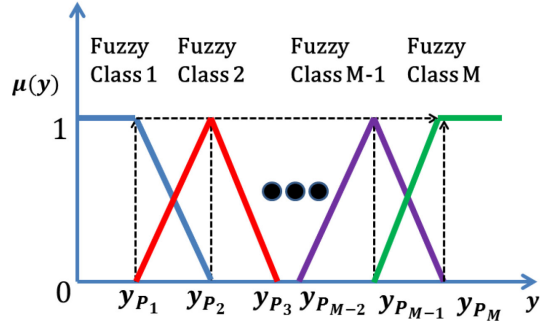

Fig. 1. 'M' fuzzy classes for training RT values using triangular fuzzy membership.

$M$ classes in a multiclass classification scenario. For each fuzzy class, a $y_{n}$ can belong to it at a membership degree $\in[0,1]$. Further, we compute an average covariance matrix for each fuzzy class as:

$$
\tilde{\boldsymbol{\Sigma}}_{m}=\frac{\sum_{n=1}^{N} \mu_{m}\left(y_{n}\right) \mathbf{X}_{n} \mathbf{X}_{n}{ }^{\top}}{\sum_{n=1}^{N} \mu_{m}\left(y_{n}\right)}, m=1, \ldots, M
$$

where $\mu_{m}\left(y_{n}\right)$ is the membership degree of $y_{n}$ in fuzzy class $m$.

We now mention the One-Versus-Rest (OVR) CSP to extend the CSP from binary classification to $M$ classes. For class $m$, OVR-CSP finds a matrix $\mathbf{W}_{m}^{*} \in \mathbb{R}^{C \times F}$, where $F$ is number of spatial filters to maximize variance of class $m$ against rest.

$$
\mathbf{W}_{m}^{*}=\underset{\mathbf{W}}{\arg \max } \frac{\operatorname{Tr}\left(\mathbf{W}^{\top} \tilde{\boldsymbol{\Sigma}}_{m} \mathbf{W}\right)}{\operatorname{Tr}\left(\mathbf{W}^{\top} \sum_{j \neq m} \tilde{\boldsymbol{\Sigma}}_{j} \mathbf{W}\right)}
$$

$\tilde{\boldsymbol{\Sigma}}_{m}$ is the mean covariance matrix of trials in class $m . \mathbf{W}_{m}^{*}$ is the concatenation of the $F$ eigenvectors associated with the $F$ largest eigenvalues of the matrix $\left(\sum_{i \neq m} \tilde{\boldsymbol{\Sigma}}_{i}\right)^{-1} \tilde{\boldsymbol{\Sigma}}_{m}$. We concatenate the obtained $F$ filters for each of the $M$ classes to obtain $\mathbf{W}^{*}=\left[\mathbf{W}_{1}^{*}, \ldots \mathbf{W}_{\mathbf{M}}^{*}\right] \in \mathbb{R}^{C \times M F}$. Then, one can compute a spatially transformed trial by $\mathbf{X}_{n}^{\prime}=\mathbf{W}^{* \top} \mathbf{X}_{n}, n=1, \ldots, N$.

\section{B. Phase Locking Value}

PLV is a statistic used to investigate synchronization of neural activity from EEG data and expresses a transient measure of connectivity. Any cognitive task results due to combination of various functional areas distributed over different regions of the brain. The task induced coupling between these areas is interpreted as synchronization of neural activity. PLV and its variants are common measures of phase synchronization.

Consider two signals $x_{1}(t)$ and $x_{2}(t)$ whose instantaneous phases are $\psi_{1}(t)$ and $\psi_{2}(t)$. In accordance with [12] the Single trial PLV (sPLV) for a given trial can be defined as follows:

$$
\operatorname{sPLV}=\left|\frac{1}{N_{s}} \sum_{t=1}^{N_{s}} e^{\left|\psi_{1}(t)-\psi_{2}(t)\right|}\right|
$$

where $N_{s}$ is the number of samples in the trial. The instantaneous phase $\psi(t)$ can be obtained using the analytic signal calculated from Hilbert transform. For any arbitrary signal $x(t)$ the analytic signal $z(t)$ is given as

$$
\begin{aligned}
& z(t)=x(t)+i \tilde{x}(t) \\
& \tilde{x}(t)=\frac{1}{\pi} \int_{-\infty}^{\infty} \frac{x(\tau)}{t-\tau} d \tau
\end{aligned}
$$

where $\tilde{x}(t)$ is the Hilbert transform of $x(t)$. The instantaneous phase $\psi(t)$ is then calculated using

$$
\psi(t)=\arctan \left(\frac{\tilde{x}(t)}{x(t)}\right)
$$

The sPLV holds a value between 0 and 1 with extremas corresponding to the cases of signal being unsynchronized and completely synchronized respectively.

\section{Differential-Phase Synchrony Representations}

Instantaneous Phase Difference (IPD) sequence $\Delta \psi(t)$ between a pair of distinct signals $s_{1}(t)$ and $s_{2}(t)$ is defined as

$$
\Delta \psi(t)=\left|\psi_{1}(t)-\psi_{2}(t)\right|
$$

Authors in [13] coupled the notion of variance of instantaneous phasors with sPLV. Furthermore, they formulated a framework to estimate a linear transform that maximizes the variance of instantaneous phasors across one class while simultaneously minimizing it across the other class. The proposed framework is similar to CSP but, in contrary, it explicitly uses phase information for binary classification. Thus, drawing an analogy from the regression CSP algorithm (Fuzzy CSPR-OVR), a novel framework is formulated to find a linear transform on the IPD sequence in such a way that it maximizes the variance across the temporal phase differences of each fuzzy class and simultaneously minimizes the variance of phases across the other fuzzy class. As the IPD sequence is used to measure synchrony between EEG signals, we refer to the extracted features as DPS representations.

1) Fuzzy Spatial Filter Optimization: Fuzzy CSPR-OVR [15] extends multiclass CSP to regression problems using fuzzy sets. Indeed, as mentioned before, the EEG signals from individual channels are prone to have a low SNR, due to spatial blurring and smearing effects. In order to obtain more discriminative DPS features for EEG classification of fuzzy classes, it thus seems prudent to compute spatial filters which maximize the variance of instantaneous phase across a particular fuzzy class and minimize across rest of them. We therefore propose an algorithm to optimize the spatial filters in order to maximize the resulting DPS feature discriminative power. Mathematically, it can be written as

$$
\mathbf{W}_{i}{ }^{*}=\underset{\mathbf{W}}{\arg \max } \frac{\operatorname{Tr}\left(\mathbf{W}^{\top} \boldsymbol{\Sigma}_{\Delta \psi_{i}} \mathbf{W}\right)}{\operatorname{Tr}\left(\mathbf{W}^{\top} \sum_{j \neq i} \boldsymbol{\Sigma}_{\Delta \psi_{j}} \mathbf{W}\right)}
$$

where $\boldsymbol{\Sigma}_{\Delta \psi_{i}}$ and $\boldsymbol{\Sigma}_{\Delta \psi_{j}}$ are the covariance matrices of the IPD sequence for the fuzzy classes $i$ and $j$. The column vectors of $\mathbf{W}$ are the spatial synchrony filters. A scheme similar to fuzzy CSP algorithm is employed for feature extraction from IPD sequence of an EEG trial in a given frequency range. The obtained features are coined as 'DPS' repersentations.

\section{EVAluATION OF DiffERENTIAL-PHASE SYNCHRONY REPRESENTATIONS FOR REACTION TIME PREDICTION}

The proposed feature representations are evaluated and compared to other phase based baseline methods on an EEG based reaction time (RT) prediction in a lane-keeping task. 
TABLE I

Number of Trials (NTRIALS) AND MEAN RT IN THE DATASET

\begin{tabular}{l|cccccc}
\hline Subject & 1 & 2 & 3 & 4 & 5 & 6 \\
\hline Ntrials & 628 & 568 & 653 & 350 & 362 & 361 \\
\hline MeanRT & 0.897 & 1.668 & 0.925 & 0.881 & 1.070 & 1.145 \\
\hline \hline Subject & 7 & 8 & 9 & 10 & 11 & 12 \\
\hline Ntrials & 239 & 484 & 550 & 641 & 190 & 143 \\
\hline MeanRT & 2.020 & 1.541 & 1.001 & 0.704 & 1.076 & 2.677
\end{tabular}

\section{A. Lane-Keeping Task}

The EEG signals were recorded from 30 active electrode sites which were placed according to modified international 10-20 electrode montage system.

The Institutional Review Board of the Veterans General Hospital, Taipei, Taiwan, approved the study. A total of 12 university students (average age 22.4, standard deviation 1.6) from the $\mathrm{Na}$ tional Chiao Tung University (NCTU) in Taiwan volunteered to support the data-collection efforts over a five-month period to study EEG correlates of attention and performance changes under specific conditions of real-world drowsiness [34].

Simulated driving experiments were conducted on a virtual reality (VR)-based dynamic driving simulator. A real car frame was mounted on a six degree-of-freedom Stewart motion platform which moved in sync with the driving scene during "motion" sessions. The motion platform was inactive during "motionless" sessions. The VR driving scene simulated nighttime cruising $(100 \mathrm{~km} / \mathrm{h})$ on a straight highway (two lanes in each direction) without other traffic. The computer program generated a random perturbation (deviation onset), and the car started to drift to the left of the right of the cruising lane with equal probability. Following each deviation, subjects were required to steer the car back to the cruising lane as quickly as possible using the steering wheel (response onset), and hold on the wheel after the car returned to the approximate center of the cruising lane (response offset). A lane departure trial is defined as consisting of three events, deviation onset, response onset, and response offset. The next lane-departure trial randomly occurs about 5 to $10 \mathrm{sec}$ after response offset in the current trial. The subjects reaction time (RT) to each lane departure trial is defined as the interval between deviation onset and response onset. If the subject does not respond promptly within 2.5 (1.5) sec, the vehicle will hit the left (right) roadside without a crash and continue to move forward against the curb event the subject completely ceases to respond. No intervention was made when the subject fell asleep and stopped responding. After reaching the lapse period, subjects resumed the task voluntarily and steered the car back to the cruising position at the earliest.

The goal is to predict RT using a 5-s EEG trial immediately before it.

\section{B. EEG Pre-Processing}

- At first, raw EEG data was passed through standard preprocessing pipeline (PREP) of EEGLAB to increase the signal to noise ratio, it comprises mainly of three operations [35] [36].
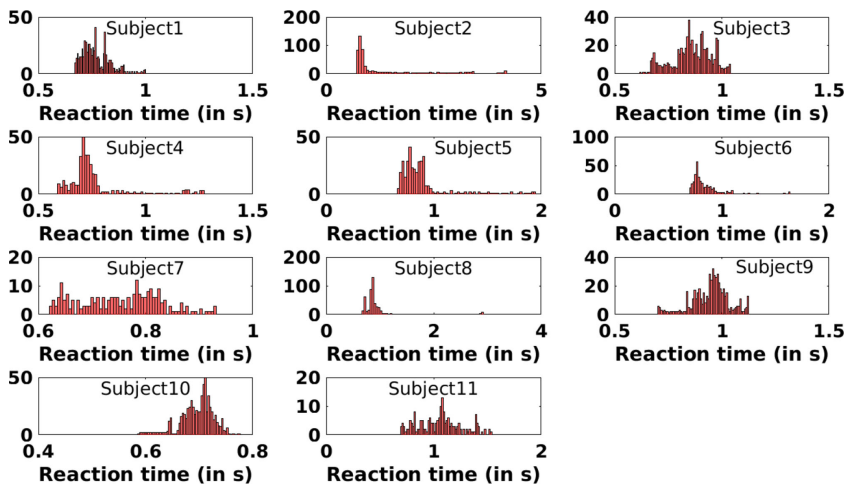

Fig. 2. Distribution of RT values.

- Removing line noise.

- Determining and removing robust reference signal.

- Interpolating the bad channels.

- Further, the data was downsampled to $250 \mathrm{~Hz}$.

- Then, the data was epoched to $5 \mathrm{sec}$ trials, i.e. if the lane deviation is starting at time ' $t$ ' then the EEG data from $[t-5, t]$ is used to predict the RT. Each EEG trial is of size $30 \times 1250$.

- Outliers in the RT values are removed by ignoring the EEG trials with RT values greater than sum of mean and three times the standard deviation.

- Thus, the obtained trials are filtered by a $[1,20] \mathrm{Hz}$ finite impulse response band-pass filter.

- The obtained data is then fed through the appropriate spatial filters.

\section{RT Pre-Processing}

The RT values for 12 subjects are pre-processed in a way similar to that of the paper (cf. section IV D of [15]). The data collected from subject 12 is erroneous with data recording anomalies and is removed from further analysis. This is because, a large number of response times were longer than 5 seconds, which are highly absurd in practice. The final distribution of RTs obtained after pre-processing are shown in Fig. 2.

\section{Feature Evaluation}

8 -fold cross-validation is used to compute the regression performance for each possible fusion of feature set and regression method. Following feature sets are extracted for each EEG trial.

1) Theta and Alpha powerband features are extracted from the band-pass filtered EEG trials. We computed the average power spectral density in the Theta band $(4-8 \mathrm{~Hz})$ and Alpha band $(8-13 \mathrm{~Hz})$ for each channel using Welchs method, and converted these $30 \times 2=60$ band powers to $\mathrm{dBs}$ as our features (denoted as ' $F S 1$ ').

2) Differential Phase Synchrony features (DPS) are extracted from the band-pass filtered EEG trials. We used 3 fuzzy sets $(K=3)$ for the RTs, and 21 spatial filters $(F=21)$ for each fuzzy class. A vector of size $(63 \times 1)=63$ constitutes the feature vector (DPS). It is denoted as ' $F S 2$ '.

3) Theta and Alpha powerband features extracted from EEG trials filtered by fuzzy CSPR-OVR. This procedure was 
TABLE II

REGRESSION PERFORMANCE OF FS1, FS2 AND FS3

\begin{tabular}{c|ccc}
\hline Feature-set & CC & RMSE & MAPE \\
\hline$F S 1$ & 0.501 & 0.0861 & 20.56 \\
\hline$F S 2$ (proposed) & 0.684 & 0.0726 & 18.02 \\
\hline$F S 3$ & 0.519 & 0.0849 & 19.30 \\
\hline$F S 4$ (proposed) & $\mathbf{0 . 7 1 9}$ & $\mathbf{0 . 0 6 9 5}$ & $\mathbf{1 6 . 4 3}$ \\
\hline
\end{tabular}

almost identical to that of ' $F S 1$ ', except that the bandpass filtered EEG trials were also spatially filtered by fuzzy CSPR-OVR before the powerband features were computed. We used 3 fuzzy sets for the RTs, and $10^{1}$ spatial filters for each fuzzy class, so that the spatially filtered EEG trials is of the dimension $30 \times 1250$, and the resultant feature ' $F S 3$ ' has $60 \times 1$ dimensions.

4) Differential Phase Synchrony features (DPS) extracted from the EEG trials pre-filtered by fuzzy CSPR-OVR. We used 3 fuzzy sets $(K=3)$ for the RTs, and 10 spatial filters $(F=10)$ for each fuzzy class again for a fair comparison. A vector of size $(63 \times 1)=63$ constitutes the feature vector (DPS-CSPR-OVR). It is denoted as ' FS4'.

All the feature sets obtained above are passed through LASSO regressor to obtain final reaction time value.

\section{E. Performance Metrics}

RMSE, CC and MAPE are the metrics in use for judging the regression performance. Assume, there are $N$ training points, $y_{d i}$ represents the true reaction time value of the $i$ th data point and $y_{i}$ represents the predicted reaction time value.

\section{F. Regression Results}

The average RMSEs, CCs and MAPEs of LASSO using the four feature sets (explained in Section III-D) are shown in the Table II. For each subject, 8-fold cross validation has been used to partition the feature set into training and validation sets. The performance is averaged across all the 8 -folds. Also, the average performance across all the subjects is reported. Here, in general 'FS4' recorded the best performance, and both FS4 and FS2 achieved much smaller RMSEs, MAPEs and much larger CCs than FS3 and FS1, suggesting that our extension of FS4 from supervised classification to supervised regression can indeed improve the regression performance.

In conclusion, FS4 had better regression performance than FS2, FS3 and FS1.

More detailed performance analysis of results and implementation details comparing performance of the features in Section III-D are presented in a supplementary file (DPSfuzzyCSPR-OVR.pdf).

\section{Multi-Task DeEP Neural Networks}

Wei et al., [37] treated drowsiness detection as a classification problem by formulating set of thresholds on reaction time

\footnotetext{
${ }^{1}$ We used 10 spatial filters here so that the filtered signals had almost the same dimensionality as the original signals, which ensured fair performance comparison.
}

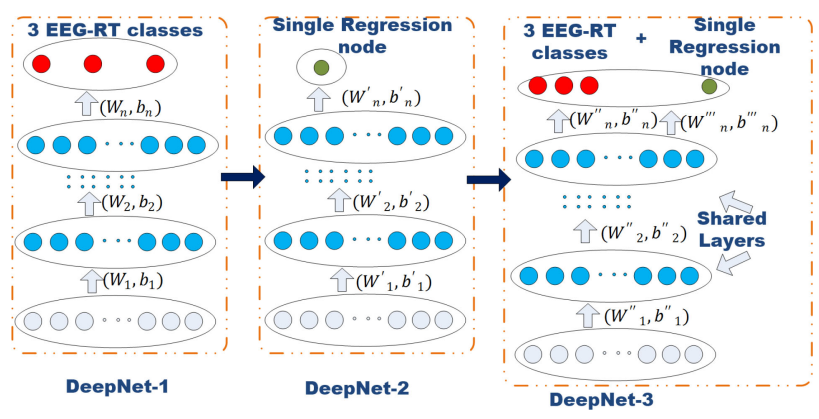

Fig. 3. Proposed Multitask-DeepNet (MTDNN) approach.

values. The ancillary task in the proposed multitask method addresses drowsiness detection as a classification problem. Two tasks in the name of primary and ancillary are in use for the experiment. EEG based RT prediction is the primary task and drowsy state classification problem is considered as an ancillary problem. As far as the ancillary task is concerned, EEG trials with $R T$ shorter than $1.5 \times($ alert $R T)$ are categorized as 'Alert' trials, whereas those with $R T$ longer than $2.5 \times($ alert $R T)$ are taken to be as 'Lapse' trials indicating 'drowsy' phase. In addition, those EEG trials with $R T$ shorter than $2.5 \times($ alert $R T)$ but longer than $1.5 \times($ alert $R T)$ are categorized as 'Semi-alert' trials. The alert $R T$ was individually estimated for each subject as suggested in [4]. Primary task is accomplished using DeepNet-2 and 3, while the ancillary task is accomplished using DeepNet-1 and 3 .

\section{A. Pre-Training}

Pre-training is used to avoid the learning algorithm to get stuck in a local optimum. This is especially true while training a deep model in the situation of a scarce training data. In the present work, we propose a supervised pre-training approach. Fig. 3 shows how the proposed Deep network-based method in DeepNet-1 and DeepNet-2 incorporates the label information. The supervised pre-training consists of two steps. Firstly, the DeepNet-1 was trained to predict the three levels of drowsy states, namely drowsy, transition and awake. Keeping the hidden layers of DeepNet-1 intact (with the pre-trained weights), an output layer consisting of a single output node is added to construct DeepNet-2, which is then further pre-trained to predict the reaction time values. Note that the regression layer of DeepNet-2 was initialized with random weights because this top layer is different from DeepNet-1. It is easier for Deep network to learn the three class problem than an infinite class classification problem (i.e. regression). Nonetheless, the weights of DeepNet-2 are tuned-up based on DeepNet-1. It follows the acumen of a meaningful human learning process: simple to complex tasks. The understanding of learning simpler tasks be able to benefit the learning for complex tasks.

\section{B. Multi-Task Objective Function}

DeepNet-1 uses the cross entropy loss-function. DeepNet-2 uses the mean-squared error loss-function. In DeepNet-1, softmax layer is the output layer, while in DeepNet-2, a sigmoid unit is present in the output. Further, the output layer of DeepNet-3 
is a composed of both softmax and sigmoid units, where the network is jointly trained for classification and regression. Accordingly, DeepNet-3 uses a combination of two losses:

$$
-\lambda \sum_{i=1}^{M} \sum_{j=1}^{N}\left(d_{i, j} \log \left(p_{i, j}\right)\right)+\mu \sum_{i=1}^{M}\left(y_{d_{i}}-y_{i}\right)^{2}
$$

In (10), $\lambda+\mu=1, M$ is the number of mini-batch samples, $N$ is the number of classes, $d_{i, j}$ represents the desired probability and $p_{i, j}$ denotes the predicted probability of the $j$ th sample for the $i$ th class. We choose $\lambda=\mu=0.5$ in our experiments.

\section{EXPERIMENTS AND RESULTS}

\section{A. Experiments on DPS-FCSPR-OVR Features From EEG RT Dataset}

The experiments are conducted on reaction time dataset for EEG Lane-keeping task. The dataset preprocessing is provided in Section III-B. $K=3$ and $F=21$ are obtained as an optimal choice for the number of fuzzy classes and the number of filters per each fuzzy class. DPS-FCSPR-OVR features are extracted from pre-processed EEG RT dataset. The dimension of the spatial filtered feature vector is $63 \times 1$. 63 dimensional feature vector is used to train DeepNet-1 with classification labels obtained by the procedure mentioned at the start of Section IV. Number of iterations used in the training of DeepNets- $\{1,2,3\}$ are $\{200,200,150\}$ respectively. Supervised pre-training steps used larger number of iterations than the fine-tuning step. Adagrad optimizer is used with the initial learning rate set to $\{0.1,0.5\}$ for supervised pre-training DeepNet- $\{1,2\}$ and joint fine-tuning DeepNet-3, respectively. We tried to avoid overfitting during training by employing drop-out (drop-out probability $p=0.5$ ) and $L_{1}$ regularization $(\lambda=0.01)$. Size of Mini-batch is set to 8. DPS-FCSPR-OVR features (input features) are normalized to zero mean and unit variance. CC, RMSE and MAPE are the metrics used for regression performance comparison, while precision, recall and F1 score are the metrics used for classification performance analysis.

\section{B. Comparison Among MTDNNs With Different Configurations}

A comparison using RMSE, CC and MAPE is being made for the multi-task deepnets with different configurations of hidden layers and number of nodes in each hidden layer. Table III contains the mean values of each of the metrics with varying number of hidden nodes and hidden layers. In figs. 4-6, an effect of the depth of the neural nets on the performance is studied. Box plots show the distribution of RMSE, CC and MAPE associated with 20 different initializations of the weights of Deepnets-1, 2 \& 3 (highest and lowest quartiles in box, plus outliers beyond highest and lowest quantiles). Mean RMSE value decreased or remained constant with increasing number of hidden layers except for a increment on transition from 5 to 6 hidden layers. A similar trend has been observed for MAPE values except that there is an increment in the MAPE value on transition from 2 to 3 hidden layers. CC strictly increased with the number of hidden layers, but it started to decrease at the transition from 5 to 6
TABLE III

REGRESSION METRICS FOR DIFFERENT ARCHITECTURES OF MTDNN

\begin{tabular}{cccc}
\hline \hline$N_{l}$ & 1 & 2 & 2 \\
\hline$N_{n}$ & {$[45]$} & {$[8050]$} & {$[10060]$} \\
\hline RMSE & 0.15 & 0.11 & 0.09 \\
\hline CC & 0.58 & 0.61 & 0.66 \\
\hline MAPE & 12.2 & 12.1 & 11.9 \\
\hline \hline$N_{l}$ & 3 & 4 & 5 \\
\hline$N_{n}$ & {$[150120$} & {$[200150$} & {$[24017013075$} \\
& $80]$ & $12045]$ & $30]$ \\
\hline RMSE & 0.06 & $\mathbf{0 . 0 5}$ & 0.06 \\
\hline CC & 0.78 & $\mathbf{0 . 8 3}$ & 0.81 \\
\hline MAPE & 11.2 & $\mathbf{9 . 7}$ & 10.5 \\
\hline \hline
\end{tabular}

$N_{l}$ : number of hidden layers; $N_{n}$ : number of hidden nodes.

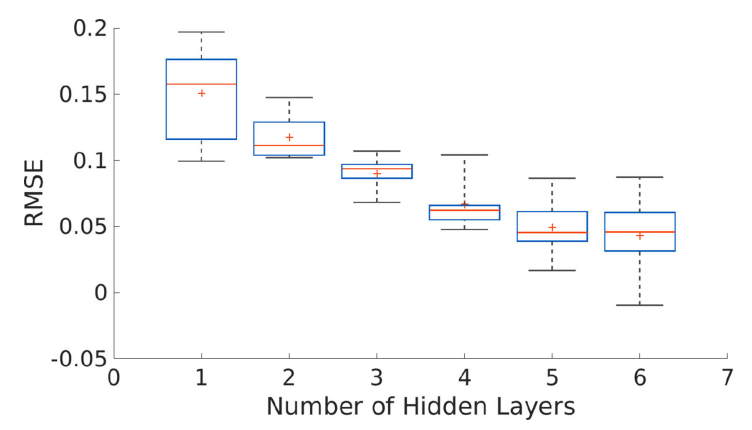

Fig. 4. Effect of changing model architecture on RMSE.

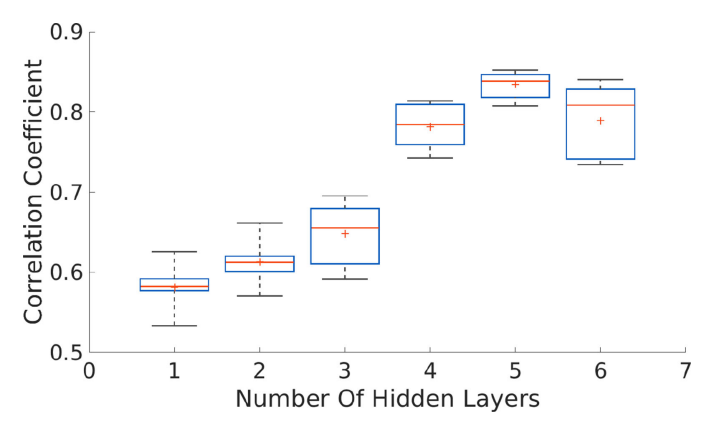

Fig. 5. Effect of changing model architecture on CC.

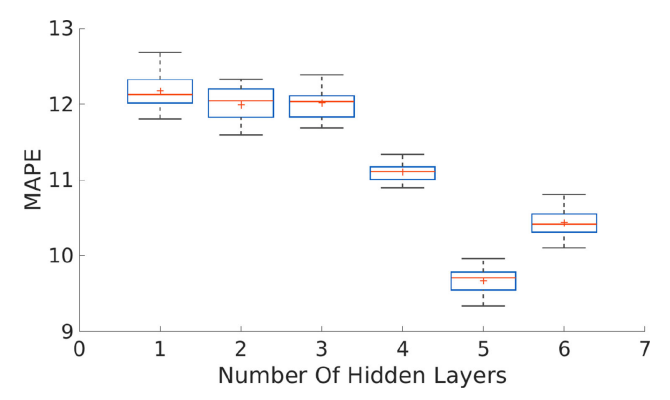

Fig. 6. Effect of changing model architecture on MAPE.

hidden layers. More number of random initialization trials are needed to make a more accurate inference. In Table III, multitask deep neural networks with $\left\{N_{n}=\left[\begin{array}{lll}150 & 120 & 80\end{array}\right], N_{l}=\right.$ $3\}$ and $\left\{N_{n}=\left[\begin{array}{llll}200 & 150 & 120 & 45\end{array}\right], N_{l}=4\right\}$ are denoted as $M T D N N_{4}$ and $M T D N N_{5}$ respectively. 
TABLE IV

COMPARISON OF REGRESSION METRIC FOR MTDNNS OVER STANDARD REGRESSION MODELS

\begin{tabular}{|c|c|c|c|c|}
\hline Model & \multicolumn{2}{|c|}{$M T D N N_{5} S V R$} & \multicolumn{2}{|c|}{$M T D N N_{4}$} \\
\hline RMSE & 0.0500 & 0.0600 & 0.0600 & \\
\hline $\mathrm{CC}$ & 0.830 & 0.778 & 0.810 & \\
\hline MAPE & 9.70 & 11.34 & 11.20 & \\
\hline Model & RR & k-NN & ANFIS & $L A S S O$ \\
\hline RMSE & 0.0700 & 0.0676 & 0.0570 & 0.0695 \\
\hline $\mathrm{CC}$ & 0.690 & 0.758 & 0.782 & 0.719 \\
\hline MAPE & 17.48 & 12.73 & 11.92 & 16.43 \\
\hline
\end{tabular}

\section{Comparison Among MTDNNs and Other Regression Models}

To further establish the powerful representational capability of the DNN-based multi-task model, a comparison with various traditional regression models like LASSO Regularized Regression, Ridge Regression (RR), Support Vector regression (SVR) [38], ANFIS (Adaptive Neuro Fuzzy Inference) ${ }^{2}$ and kNN (knearest neighbours) is investigated. LASSO and Ridge regression models are implemented in Matlab R2016. We utilized scikit-learn SVR tool [39] and scikit-learn respectively to run the SVR and kNN simulations. Based on the simulations, we observe that: a) SVR is poor at dealing with large scale training datasets due to the computational complexity and memory storage; b) with a high input feature dimension, the generalization ability of SVR is very limited [40]. High computational resources are associated with solving quadratic programming problem amid the optimization of the cost function in the training stage of the SVM. Additionally, a requirement for a large memory to store the kernel products also limits the pertinence of using the SVMs on very large data sets. Grid search technique is employed to determine parameters $C$ and $\gamma$, which are set as $2^{5}$ and $2^{2}$, respectively. $\epsilon$ is set to 0.2 . For the two regression methods: LASSO and RR, the adjustable parameter $\lambda$ was optimized by an inner 8-fold cross-validation [41] on the training dataset. We employ $k=5$ with inverse distance weighting for $\mathrm{kNN}$ regression. Feature vector of 63 dimensions is used to train the ANFIS regressor with 5 fuzzy partitions for each dimension. Gaussian and linear memberships are chosen for input and output memberships respectively.

On an average, from Table IV, the proposed $M T D N N_{4}$ and $M T D N N_{5}$ approaches clearly outperformed SVR, LASSO, RR, kNN and ANFIS schemes in-terms of RMSE, CC and MAPE. In all these experiments, we used $M T D N N_{5}$ (from Table III) as the representative MTDNN model. Each of the MTDNN is trained for 100 epochs with a learning rate of 0.1 using Adagrad.

The respective percentage performance improvements of MTDNN over the other regression models are shown in the Figs. 7-9. For instance, the terms in legend 'MTDNN/SVR' represent the improvements with MTDNN over the SVR method. The notation of other terms in the legend is to be understood in a similar manner. On an average, MTDNN recorded a $15.49 \%$

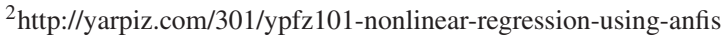

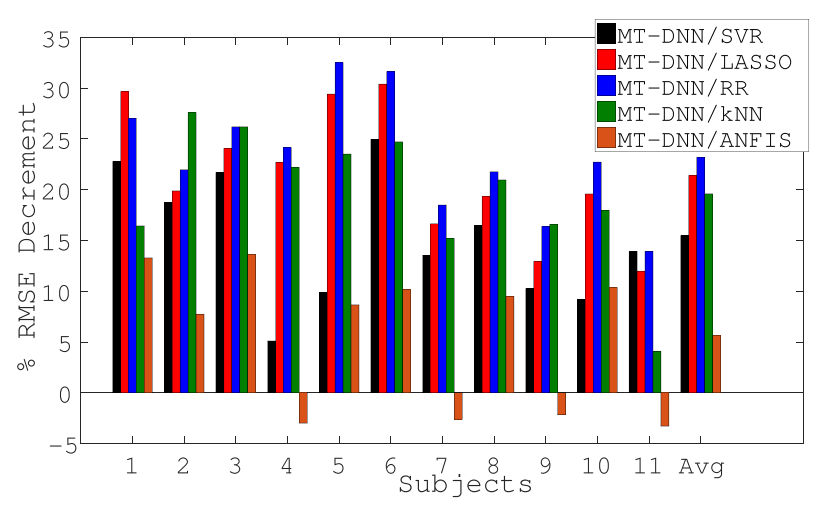

Fig. 7. Percent improvement (decrement) in RMSE with proposed MTDNN over other methods.

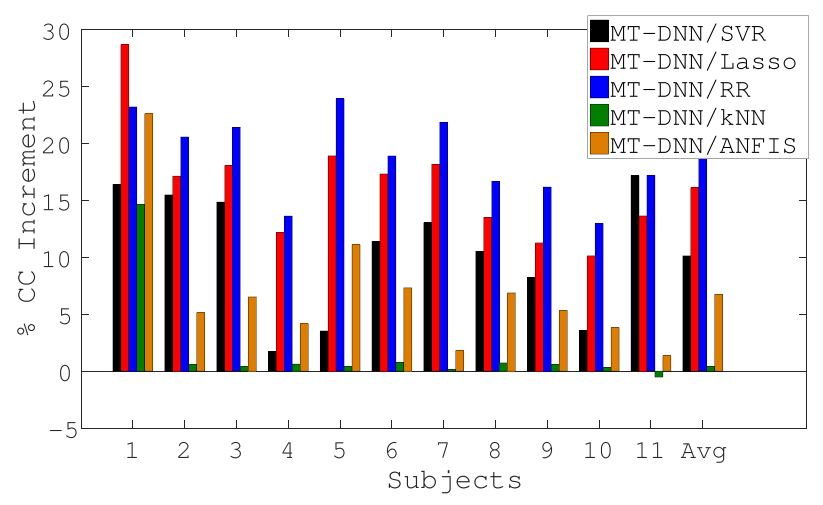

Fig. 8. Percent improvement (increment) in CC with proposed MTDNN over other methods.

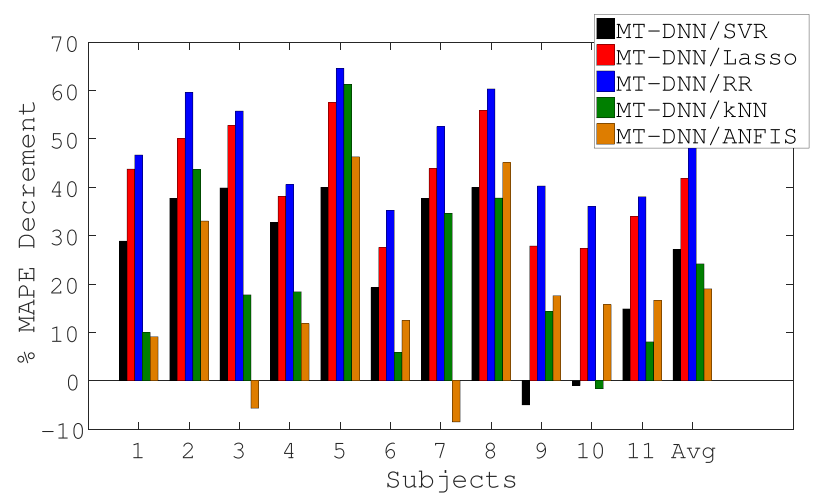

Fig. 9. Percent improvement (decrement) in MAPE with proposed MTDNN over other methods.

TABLE V

TWO-WAY ANOVA RESULTS MTDNN VERSUS OTHERS

\begin{tabular}{c|ccc}
\hline & RMSE & CC & MAPE \\
\hline$p-$ value & 0.015 & $\mathbf{0 . 0 0 0}$ & 0.002 \\
\hline
\end{tabular}

smaller RMSE, a $27.15 \%$ smaller MAPE and a $10.13 \%$ larger CC than SVR. On an average, MTDNN had performed with a $21.41 \%$ smaller RMSE, a $41.81 \%$ smaller MAPE and a $16.11 \%$ larger CC than LASSO. Also, MTDNN had performed with a $23.18 \%$ smaller RMSE, a $18.67 \%$ smaller MAPE and a $48.17 \%$ larger CC than RR. In a similar manner, on an average, MTDNN 
TABLE VI

Non-Parametric Multiple Comparison Tests Based on Paired T-Tests (MTDNNs Versus Other Regression Models) MTDNN Denoted by $M$

\begin{tabular}{c|cccc|ccccc|cccc|}
\hline & \multicolumn{4}{|c}{ RMSE } & \multicolumn{1}{c|}{ CC } & \multicolumn{4}{c|}{ MAPE } \\
\hline & $M$ & SVR & LASSO & ANFIS & $M$ & SVR & LASSO & ANFIS & $M$ & SVR & LASSO & ANFIS \\
\hline$M$ & & 0.02 & $\mathbf{0 . 0 0 1}$ & 0.01 & \multicolumn{3}{c|}{$\mathbf{0 . 0 0 0}$} & 0.002 & 0.042 & & 0.01 & 0.01 & 0.01 \\
\hline RR & 0.03 & 0.04 & 0.02 & 0.04 & 0.03 & 0.01 & $\mathbf{0 . 0 0 0}$ & 0.01 & $\mathbf{0 . 0 0 0}$ & 0.01 & 0.01 & 0.03 \\
\hline SVR & 0.02 & & $\mathbf{0 . 0 0 1}$ & 0.02 & $\mathbf{0 . 0 0 0}$ & 0.02 & 0.03 & 0.01 & $\mathbf{0 . 0 0 0}$ & 0.023 \\
\hline kNN & 0.02 & 0.024 & $\mathbf{0 . 0 0 1}$ & $\mathbf{0 . 0 0 0}$ & $\mathbf{0 . 0 0 0} 0.004$ & 0.02 & 0.045 & 0.02 & 0.01 & 0.034 & $\mathbf{0 . 0 0 0}$ \\
\hline
\end{tabular}

outperformed ANFIS and kNN approaches with a 5.66\% and $19.59 \%$ smaller RMSE, $18.98 \%$ and $24.16 \%$ smaller MAPE, $6.76 \%$ and $0.5 \%$ larger $\mathrm{CC}$ values respectively. Performance across subjects 6, 7 and 10 needs to be analyzed further while collecting more EEG sessions from these subjects, so that the $M T D N N$ can be trained sufficiently for better inference.

Further, Statistical analysis is performed to test several hypotheses on MAPE, RMSE and CC's across the subjects. A two-way Analysis of Variance (ANOVA) is performed for different types of regression schemes with the objective to figure out if the RMSE, CC and MAPE variations due to the changes in regressors are statistically significant, with the subjects treated as a random factor. The results are shown in Table V, $(p-$ value $<0.05)$ which indicates that there were statistically significant differences in RMSEs, CCs and MAPEs among different regression methods with subjects as a random factor. In other words, there is a significant main effect of regression method on the performance metrics RMSE, CC, MAPE $(p-$ value $<0.05$, cf. Table V).

Then, post-hoc non-parametric multiple comparison tests (paired t-tests in this case) are conducted to find out if the difference between pair of regressors is statistically significant, with the $p$-value corrected employing the False Discovery Rate method [42]. The p-values are shown in Table VI, where in most of the values are statistically significant. The bolded ones in Table VI are extremely statistically significant. ${ }^{3}$

\section{Comparison Among DNNs and MTDNNs}

In this section, we compare the performance of the Multitask DNNs $\left(M T D N N_{4}\right.$ and $\left.M T D N N_{5}\right)$ with vanilla DNNs (4 hidden layer architecture of $M T D N N_{5}\left(V D N_{5}\right)$ and 3 hidden layer architecture of $M T D N N_{4}\left(V D N_{4}\right)$ respectively). Table VII presents a systematic performance comparison on the basis of several regression metrics. Each of the MTDNNs and vanilla DNNs ( $V D N_{4}$ and $V D N_{5}$ ) are trained for 100 epochs with a learning rate of 0.1 using Adagrad. We circumvent overfitting during training by using drop-out (drop-out probability $p=0.5$ ) and $L_{1}$ regularization $(\lambda=0.01$ ). Fig. 13 presents the average generalization ability of the proposed $M T D N N$ approach for a stable [43] [44] learning rate $\eta$. One can infer that, on an average, the network is not over-fitting. The respective percentage performance improvements of MTDNNs over the conventional or vanilla DNN regression models are shown in the figs. 10-12. For example, the terms in legend

${ }^{3} p-$ value $\leq 0.001$.
TABLE VII

REGRESSION METRICS FOR MTDNNS AND CORRESPONDING VDNS WITH SAME HIDDEN LAYER CONFIGURATION

\begin{tabular}{ccccc}
\hline Model & $M T D N N_{4}$ & $V D N_{4}$ & $M T D N N_{5}$ & $V D N_{5}$ \\
\hline$N_{l}$ & 3 & 3 & 4 & 4 \\
\hline$N_{n}$ & {$[150120$} & {$[150120$} & {$[200150$} & {$[200150$} \\
& $80]$ & $80]$ & $12045]$ & $12045]$ \\
\hline RMSE & 0.06 & 0.08 & $\mathbf{0 . 0 5}$ & 0.07 \\
\hline CC & $\mathbf{0 . 7 8}$ & 0.64 & 0.83 & 0.69 \\
\hline MAPE & 11.2 & 14.8 & $\mathbf{9 . 7}$ & 17.5 \\
\hline
\end{tabular}

$N_{l}$ : number of hidden layers, $N_{n}$ : number of hidden nodes.

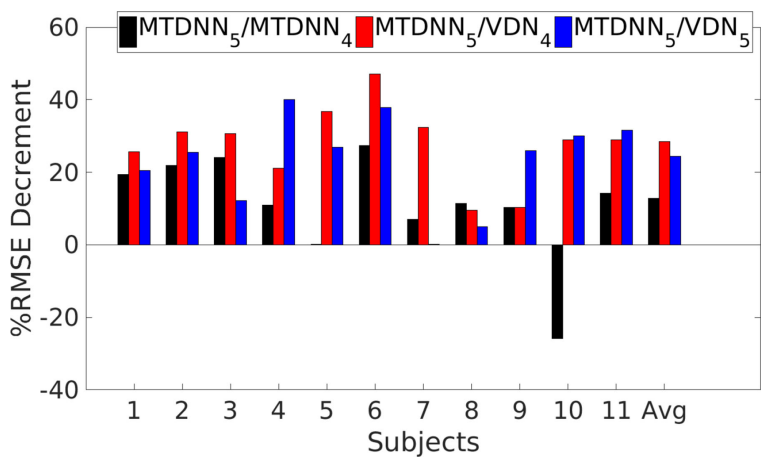

Fig. 10. Percent improvement (decrement) in RMSE with proposed MTDNN over other DNNs.

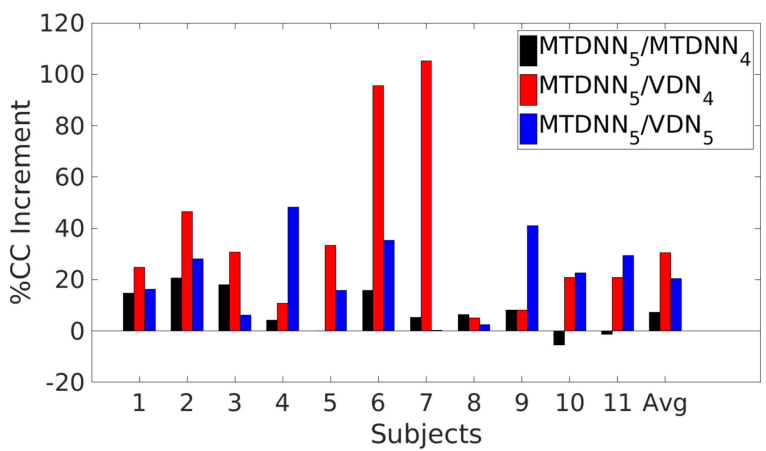

Fig. 11. Percent improvement (increment) in CC with proposed MTDNN over other DNNs.

$M T D N N_{5} / V D N_{5}$ represent the improvements with Multitask DNN $\left(M T D N N_{5}\right)$ over the vanilla DNN $\left(V D N_{5}\right)$ method. The notation of other terms in the legend is to be understood in a similar manner. On an average, $M T D N N_{5}$ recorded a $12.88 \%$ smaller RMSE, a $7.28 \%$ larger CC and a $14.83 \%$ smaller MAPE than $M T D N N_{4}$. On an average, $M T D N N_{5}$ had performed with a $28.53 \%$ smaller RMSE, a $35.20 \%$ smaller MAPE and 


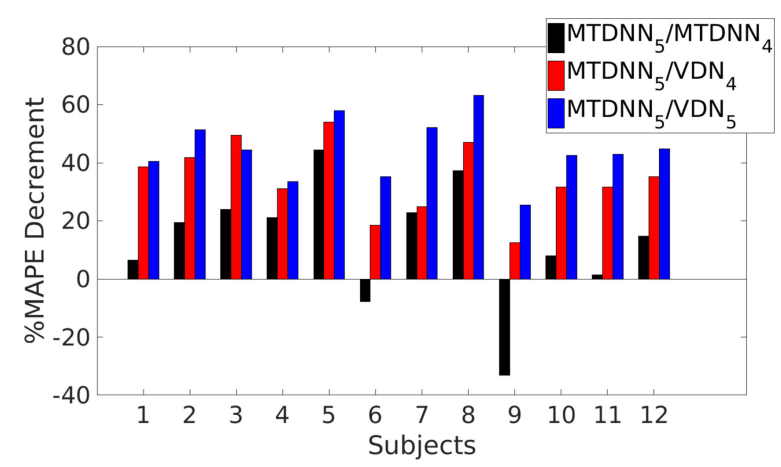

Fig. 12. Percent improvement (decrement) in MAPE with proposed MTDNN over other DNNs.

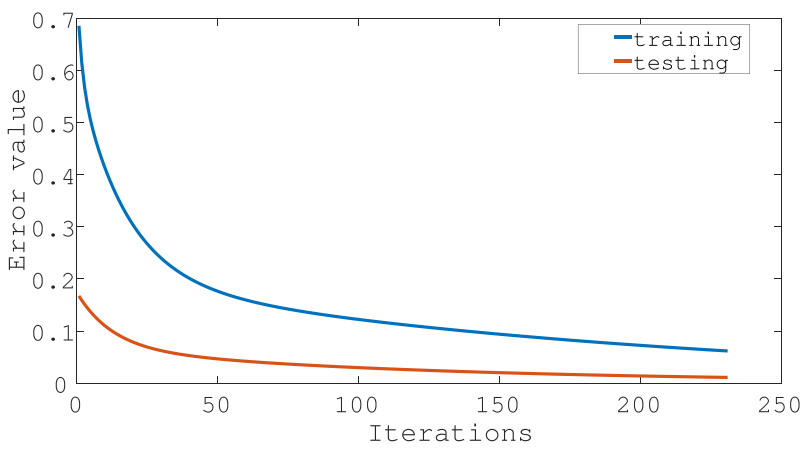

Fig. 13. Average Training and generalization error for proposed MTDNN.

TABLE VIII

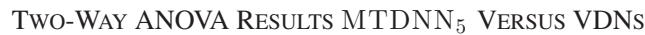

\begin{tabular}{c|ccc}
\hline & RMSE & CC & MAPE \\
\hline$p-$ value & 0.005 & $\mathbf{0 . 0 0 0}$ & $\mathbf{0 . 0 0 0}$ \\
\hline
\end{tabular}

a $30.42 \%$ larger CC than $V D N_{4}$. Also, $M T D N N_{5}$ had performed with a $24.4 \%$ smaller RMSE, a $44.70 \%$ smaller MAPE and a $20.51 \%$ larger CC than $V D N_{5}$.

Further, Statistical analysis is performed to test several hypotheses on MAPE, RMSE and CC's across the subjects. Firstly, a two-way Analysis of Variance (ANOVA) is performed for various MTDNNs and VDN's with the intent to assess if the RMSE, CC and MAPE discrepancies due to the changes in the factor $(M T D N N / V D N)$ are statistically significant, with the subjects set as a random factor. The results are shown in Table VIII, $(p-$ value $<0.05)$ which indicates that there were statistically significant differences in RMSEs, CCs and MAPEs among MTDNNs and VDNs with subjects regarded as a random factor. In other words, there is a significant main effect of the type of DNNs (Vanilla or Multitask) on the performance metrics RMSE, CC, MAPE ( $p$ - value $<0.05$, cf. Table VIII).

In addition, post-hoc non-parametric multiple comparison tests (paired t-tests in this case) are conducted to find out if the difference between multitask and vanilla modules is statistically significant, with the $p$-value corrected employing the False Discovery Rate method [42]. The p-values are shown in Table IX, where in the values are statistically significant. In all the pairwise comparisons $M T D N_{5} / V D N_{4}$, $M T D N N_{5} / V D N_{5}$ etc., the results are statistically signifi-
TABLE IX

Non-Parametric Multiple COMPARISON Tests Based on Paired T-Tests (MTDNNS VERSUS OTHER DNNS), MTDNN AND VDN DENOTED BY $M$ AND $V$ RESP

\begin{tabular}{c|ccc|ccc|ccc}
\hline & \multicolumn{3}{|c}{ RMSE } & \multicolumn{4}{c}{ CC } & \multicolumn{3}{c}{ MAPE } \\
\hline & $M_{5}$ & $V_{4}$ & $V_{5}$ & $M_{5}$ & $V_{4}$ & $V_{5}$ & $M_{5}$ & $V_{4}$ & $V_{5}$ \\
\hline$M_{5}$ & & 0.02 & $\mathbf{0 . 0 0 1}$ & & 0.01 & $\mathbf{0 . 0 0 0}$ & & $\mathbf{0 . 0 0 0}$ & 0.01 \\
\hline$V_{4}$ & 0.02 & 0.04 & 0.02 & 0.01 & 0.01 & $\mathbf{0 . 0 0 0}$ & $\mathbf{0 . 0 0 0}$ & 0.01 & 0.01 \\
\hline$V_{5}$ & $\mathbf{0 . 0 0 1}$ & 0.02 & $\mathbf{0 . 0 0 1}$ & $\mathbf{0 . 0 0 0}$ & $\mathbf{0 . 0 0 0}$ & 0.02 & 0.01 & 0.01 & $\mathbf{0 . 0 0 0}$ \\
\hline
\end{tabular}

TABLE X

Classification (Auxiliary Task) Performance of Proposed Method, AVERAGED OVER All SUbJECTS

\begin{tabular}{c|ccc|ccc}
\hline & \multicolumn{3}{|c|}{$M T D N N_{4}$} & \multicolumn{3}{c}{$M T D N N_{5}$} \\
\hline Class & Alert & Semi-alert & Lapse & Alert & Semi-alert & Lapse \\
\hline \hline Precision & 0.91 & 0.90 & 0.94 & 0.90 & 0.92 & 0.92 \\
\hline Recall & 0.88 & 0.89 & 0.91 & 0.91 & 0.93 & 0.94 \\
\hline F1 & 0.89 & 0.89 & 0.92 & 0.90 & 0.92 & 0.93 \\
\hline
\end{tabular}

cant. The bolded ones in Table IX are extremely statistically significant. ${ }^{4}$

\section{E. Classification Results for the Ancillary Task for the Proposed MTDNNs}

Table $\mathrm{X}$ contains the classification performance of the proposed MTDNNs for the ancillary task (drowsiness classification). Since this is an ancillary task, we have not provided a detailed comparison with baseline methods.

\section{DISCUSSION}

In this work, we propose the Differential Phase Synchrony (DPS) representations for regression. Moreover, we integrate it with fuzzy-CSPOVR framework (DPS-FCSPR-OVR). We further validate the proposed features on the RT dataset from an EEG based lane-keeping task. 8-fold cross validation is used for all the experiments. Statistical analysis and regression results validate the effectiveness of proposed DPS-FCSPR-OVR representations. An interesting direction of future work is to integrate DPS features with Regularised Fuzzy-CSP and SFBCSP. In future, we seek to integrate IPD sequence with CSP through JAD (Joint Approximate Diagonalization) framework. In addition, MTDNN based regressor is proposed to predict the reaction time with an ancillary DNN trained for drowsiness prediction dealt as a classification problem. Multitask learning enforces regularization hence reduces the risk of overfitting, and hence an improved performance. Furthermore, as both of tasks are related tasks and complimentary to each other, multitask learning bolster the performance in comparison to either of the tasks. A computational complexity analysis of proposed approach is included in a supplementary file for the readers. Due to the non-availability of a public dataset for regression, In our research, we majorly focus on our driving data, instead of the other data.

\footnotetext{
${ }^{4} p-$ value $\leq 0.001$
} 


\section{CONCLUSION}

In this paper, by drawing an analogy with the regression CSP algorithm (Fuzzy-CSPOVR), a novel framework is formulated to find a linear transform on the IPD sequence in such a way that it maximizes the variance across the temporal phase differences of each fuzzy class and simultaneously minimizes the variance of phases across the other fuzzy class. The IPD sequence is used to quantify synchrony between EEG signals. We refer to the extracted features as Differential-phase synchrony (DPS) representations. Proposed DPS feature framework performed superior to powerband features in-terms of better RMSE, Correlation Coefficient (CC) and MAPE values when both DPS and powerband features are passed through LASSO. In addition, a Multitask DeepNet (MTDNN) approach is proposed, where, a three step procedure is used to train on both drowsiness classification and RT prediction tasks. A comparison is made among different configurations of MTDNNs. Also, MTDNNs are compared with single task DNNs, SVR (Support Vector Regression), LASSO, RR (Ridge Regression), kNN and ANFIS. The proposed MTDNN (MTDNN $N_{5}$ ) exhibited a $15.49 \%$ smaller RMSE, a 27.15\% smaller MAPE and a $10.13 \%$ larger CC than SVR. The proposed approach achieved the best performance especially suggesting its utility in BCI applications of online adaptation with small training data. Also, the present study is a first step towards realizing large scale Multi-task learning BCIs. The two most prominent pre-training approaches for DNN's are the RBM [45] and stacked auto-encoder [46] algorithms. But, both of the above algorithms are unsupervised. Semi-supervised multi-task approaches can be more advantageous and will be explored in a future work.

\section{REFERENCES}

[1] T. Toroyan, M. M. Peden, and K. Iaych, "WHO launches second global status report on road safety," Inj. Prev., vol. 19, no. 2, p. 150, 2013.

[2] S. K. Lal and A. Craig, "A critical review of the psychophysiology of driver fatigue," Biol. Psychol., vol. 55, no. 3, pp. 173-194, 2001.

[3] B. T. Jap, S. Lal, P. Fischer, and E. Bekiaris, "Using EEG spectral components to assess algorithms for detecting fatigue," Expert Syst. Appl., vol. 36, no. 2, pp. 2352-2359, 2009.

[4] C.-S. Wei, Y.-P. Lin, Y.-T. Wang, T.-P. Jung, N. Bigdely-Shamlo, and C.-T. Lin, "Selective transfer learning for EEG-based drowsiness detection," in Proc. IEEE Int. Conf. Syst. Man Cybern., 2015, pp. 3229-3232.

[5] G. N. Dimitrakopoulos, I. Kakkos, N. V. Thakor, A. Bezerianos, and Y. Sun, "A mental fatigue index based on regression using multiband EEG features with application in simulated driving," in Proc. IEEE 39th Annu. Int. Conf. Eng. Med. Biol. Soc., 2017, pp. 3220-3223.

[6] H. Wang, A. Dragomir, N. I. Abbasi, J. Li, N. V. Thakor, and A. Bezerianos, "A novel real-time driving fatigue detection system based on wireless dry EEG," Cogn. Neurodynamics, vol. 12, pp. 365-376, 2018.

[7] A. Saha, A. Konar, and A. K. Nagar, "EEG analysis for cognitive failure detection in driving using type-2 fuzzy classifiers," IEEE Trans. Emerg. Topics Comput. Intell., vol. 1, no. 6, pp. 437-453, Dec. 2017.

[8] A. Pikovsky, M. Rosenblum, and J. Kurths, Synchronization: A Universal Concept in Nonlinear Sciences, vol. 12. Cambridge, U.K.: Cambridge Univ. Press, 2003.

[9] J. Qu, R. Wang, C. Yan, and Y. Du, "Oscillations and synchrony in a cortical neural network," Cogn. Neurodynamics, vol. 8, no. 2, pp. 157166, 2014.

[10] J.-P. Lachaux, E. Rodriguez, J. Martinerie, and F. J. Varela, "Measuring phase synchrony in brain signals," Human Brain Mapping, vol. 8, no. 4, pp. 194-208, 1999.

[11] J.-P. Lachaux, E. Rodriguez, M. Le Van Quyen, A. Lutz, J. Martinerie, and F. J. Varela, "Studying single-trials of phase synchronous activity in the brain," Int. J. Bifurcation Chaos, vol. 10, no. 10, pp. 2429-2439, 2000.
[12] N. Caramia, F. Lotte, and S. Ramat, "Optimizing spatial filter pairs for EEG classification based on phase-synchronization," in Proc. IEEE Int. Conf. Acoust. Speech Signal Process., 2014, pp. 2049-2053.

[13] S. Kumar, T. K. Reddy, and L. Behera, "EEG based motor imagery classification using instantaneous phase difference sequence," in Proc. IEEE Int. Conf. Syst. Man Cybern., 2018, to be published.

[14] W. Kong, Z. Zhou, B. Jiang, F. Babiloni, and G. Borghini, "Assessment of driving fatigue based on intra/inter-region phase synchronization," Neurocomputing, vol. 219, pp. 474-482, 2017.

[15] D. Wu, J.-T. King, C.-H. Chuang, C.-T. Lin, and T.-P. Jung, "Spatial filtering for EEG-based regression problems in brain-computer interface (BCI)," IEEE Trans. Fuzzy Syst., vol. 26, no. 2, pp. 771-781, Apr. 2018.

[16] J. Frey, M. Daniel, J. Castet, M. Hachet, and F. Lotte, "Framework for electroencephalography-based evaluation of user experience," in Proc. CHI Conf. Human Factors Comput. Syst., 2016, pp. 2283-2294.

[17] A. Meinel, S. Casta no-Candamil, J. Reis, and M. Tangermann, "Pre-trial EEG-based single-trial motor performance prediction to enhance neuroergonomics for a hand force task," Front. Hum. Neurosci., vol. 10, p. 170, 2016.

[18] D. Wu, B. J. Lance, V. J. Lawhern, S. Gordon, T.-P. Jung, and C.-T. Lin, "EEG-based user reaction time estimation using riemannian geometry features," IEEE Trans. Neural Syst. Rehabil. Eng., vol. 25, no. 11, pp. 2157-2168, Nov. 2017.

[19] A. Meinel, F. Lotte, and M. Tangermann, "Tikhonov regularization enhances EEG-based spatial filtering for single trial regression," in Proc. Int. Brain-Comput. Interface Conf., 2017, pp. 1-6.

[20] T. K. Reddy, V. Arora, L. Behera, Y. Wang, and C. T. Lin, "Multi-class fuzzy time-delay common spatio-spectral patterns with fuzzy information theoretic optimization for EEG-based regression problems in braincomputer interface (BCI)," IEEE Trans. Fuzzy Syst., to be published.

[21] R. Frömer, M. Maier, and R. Abdel Rahman, "Group-level EEGprocessing pipeline for flexible single trial-based analyses including linear mixed models," Front. Neurosci., vol. 12, p. 48, 2018.

[22] I. Sturm, S. Dähne, B. Blankertz, and G. Curio, "Multi-variate EEG analysis as a novel tool to examine brain responses to naturalistic music stimuli," PloS One, vol. 10, no. 10, p. e0141281, 2015.

[23] Y.-T. Liu, Y.-Y. Lin, S.-L. Wu, C.-H. Chuang, and C.-T. Lin, "Brain dynamics in predicting driving fatigue using a recurrent self-evolving fuzzy neural network," IEEE Trans. Neural Netw. Learn. Syst., vol. 27, no. 2, pp. 347-360, Feb. 2016.

[24] D. Wu, V. J. Lawhern, S. Gordon, B. J. Lance, and C.-T. Lin, "Driver drowsiness estimation from EEG signals using online weighted adaptation regularization for regression (OwARR)," IEEE Trans. Fuzzy Syst., vol. 25, no. 6, Dec. 2017.

[25] L. Xie, Z. Deng, P. Xu, K.-S. Choi, and S. Wang, "Generalized hidden-mapping transductive transfer learning for recognition of epileptic electroencephalogram signals," IEEE Trans. Cybern, to be published, doi: 10.1109/TCYB.2018.2821764.

[26] D. Wu, "Pool-based sequential active learning for regression," 2018, arXiv: 1805.04735 .

[27] H. Zuo, G. Zhang, W. Pedrycz, V. Behbood, and J. Lu, "Granular fuzzy regression domain adaptation in takagi-sugeno fuzzy models," IEEE Trans. Fuzzy Syst., vol. 26, no. 2, pp. 847-858, Apr. 2018.

[28] S. Sun, X. Xie, and C. Dong, "Multiview learning with generalized eigenvalue proximal support vector machines," IEEE Trans. Cybern, to be published, doi: 10.1109/TCYB.2017.2786719.

[29] V. Jayaram, M. Alamgir, Y. Altun, B. Scholkopf, and M. Grosse-Wentrup, "Transfer learning in brain-computer interfaces," IEEE Comput. Intell. Mag., vol. 11, no. 1, pp. 20-31, Feb. 2016.

[30] Y. Zhang and Q. Yang, "A survey on multi-task learning," 2017, arXiv: 1707.08114 .

[31] F. Lotte et al., "A review of classification algorithms for EEG-based brain-computer interfaces: A 10 year update," J. Neural Eng., vol. 15, no. 3, 2018, Art. no. 031005 .

[32] M. Alamgir, M. Grosse-Wentrup, and Y. Altun, "Multitask learning for brain-computer interfaces," in Proc. 13th Int. Conf. Artif. Intell. Statist., 2010, pp. 17-24.

[33] C.-H. Chuang, L.-W. Ko, T.-P. Jung, and C.-T. Lin, "Kinesthesia in a sustained-attention driving task," Neuroimage, vol. 91, pp. 187-202, 2014.

[34] S. Kerick et al., "Inter-and intra-individual variations in sleep, subjective fatigue, and vigilance task performance of students in their real-world environments over extended periods," submitted for publication.

[35] N. Bigdely-Shamlo, T. Mullen, C. Kothe, K.-M. Su, and K. A. Robbins, "The PREP pipeline: Standardized preprocessing for large-scale EEG analysis," Front. Neuroinform., vol. 9, p. 16, 2015. 
[36] EEG-Clean-Tools (PREP Pipeline). [Online]. Available: http://vislab. github.io/EEG-Clean-Tools/

[37] C.-S. Wei, Y.-P. Lin, Y.-T. Wang, C. Lin, and T.-P. Jung, "Transfer learning with large-scale data in brain-computer interfaces," in Proc. IEEE Annu. Int. Conf. Eng. Med. Biol. Soc., 2016, pp. 4666-4669.

[38] M. Sánchez-Fernández, M. de Prado-Cumplido, J. Arenas-García, and F. Pérez-Cruz, "SVM multi-regression for nonlinear channel estimation in multiple-input multiple-output systems," IEEE Trans. Signal Process., vol. 52, no. 8, pp. 2298-2307, Aug. 2004.

[39] F. Pedregosa et al., "Scikit-learn: Machine learning in python," J. Mach. Learn. Res., vol. 12, pp. 2825-2830, 2011.

[40] I. Kondofersky and F. J. Theis, "TREVOR HASTIE, ROBERT TIBSHIRANI, and MARTIN WAINWRIGHT. Statistical learning with sparsity: The lasso and generalizations. Boca Raton: CRC press," Biometrics, vol. 74, no. 2, pp. 769-769, 2018.

[41] G. C. Cawley and N. L. Talbot, "On over-fitting in model selection and subsequent selection bias in performance evaluation," J. Mach. Learn. Res., vol. 11, no. Jul, pp. 2079-2107, 2010.

[42] Y. Benjamini and Y. Hochberg, "Controlling the false discovery rate: A practical and powerful approach to multiple testing," J. Roy. Stat. Soc. Ser. B (Methodological), vol. 57, pp. 289-300, 1995.

[43] O. Bousquet and A. Elisseeff, "Stability and generalization," J. Mach. Learn. Res., vol. 2, no. Mar., pp. 499-526, 2002.

[44] T. K. Reddy, V. Arora, and L. Behera, "HJB-equation-based optimal learning scheme for neural networks with applications in brain-computer interface," IEEE Trans. Emerg. Topics Comput. Intell, to be published, doi: 10.1109/TETCI.2018.2858761.

[45] T. K. Reddy and L. Behera, "Online eye state recognition from EEG data using deep architectures," in Proc. IEEE Int. Conf. Syst. Man Cybern., 2016, pp. $000712-000717$.

[46] A. Majumder, L. Behera, and V. K. Subramanian, "Automatic facial expression recognition system using deep network-based data fusion," IEEE Trans. Cybern., vol. 48, no. 1, pp. 103-114, Jan. 2018.

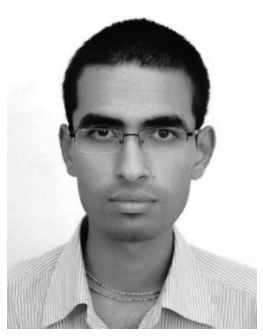

Tharun Kumar Reddy received the B.Tech. degree in electrical engineering from the Indian Institute of Technology Kanpur, Kanpur, India, where he is currently working toward the Ph.D. degree. His research interests include deep learning for brain-computer interfaces, multimodal biosignal processing, machine learning, and biomedical signal processing.

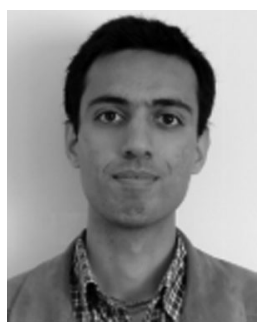

Vipul Arora received the B.Tech. and Ph.D. degrees in electrical engineering from the Indian Institute of Technology Kanpur (IIT), Kanpur, India. He was a Research Scientist with the Amazon Alexa Team, Boston, MA, USA. He is currently an Assistant Professor with the Department Of Electrical Engineering, IIT Kanpur, Kanpur, India. His research interests include music information retrieval, acoustic space modeling and semantic signal processing.

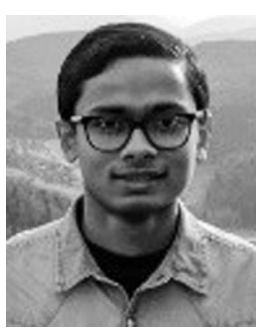

Satyam Kumar received the B.Tech. and M. Tech. degrees in electrical engineering from the Indian Institute of Technology Kanpur, Kanpur, India. His research interests include brain-computer interfaces, machine learning, biomedical signal processing, and Riemannian geometry applied to robust signal processing.

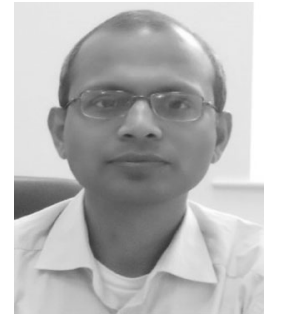

Laxmidhar Behera (S'92-M'03-SM'03) received the B.Sc. and M.Sc. degrees in engineering from National Institute of Technology Rourkela, Rourkela, India, in 1988 and 1990, respectively. He received the $\mathrm{Ph} . \mathrm{D}$. degree from Indian Institute of Technology Delhi, Delhi, India, in 1996. He is currently working as a Professor with the Department of Electrical Engineering, Indian Institute of Technology Kanpur, Kanpur, India. He is also a Guest Professor with Hangzhou Dinazi University, Hangzhou, China. He has worked as an Assistant Professor with Birla Institute of Technology and Science, Pilani during 1995-1999 and pursued the postdoctoral studies with the German National Research Center for Information Technology, Sank Augustin, Germany, during 2000-2001. He worked as a Reader with Intelligent Systems Research Center, University of Ulster, United Kingdom during 2008-2010. He has also worked as a Visiting Researcher/Professor with FHG, Germany, and ETH, Zurich, Switzerland. He has more than 200 papers to his credit published in refereed journals and conference proceedings. His research interests include intelligent control, robotics, semantic signal/music processing, neural networks, control of cyber-physical systems and cognitive modeling. He is Fellow of Indian National Academy of Engineering.

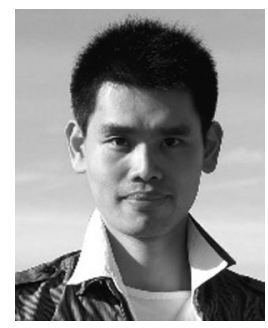

Yu-Kai Wang (M'13) received the B.S. degree in mathematics education from the National Taichung University of Education, Taichung, Taiwan, in 2006 , the M.S. degree in biomedical engineering from $\mathrm{Na}-$ tional Chiao Tung University (NCTU), Hsinchu, Taiwan, in 2009, and the Ph.D. degree in computer science from NCTU, Hsinchu, Taiwan, in 2015. He was a Visiting Scholar with the Swartz Center for Computational Neuroscience, University of California at San Diego, La Jolla, CA, USA, from 2013 to 2014. He is currently a Lecturer with the University of Technology Sydney, Ultimo, NSW, Australia. His current research interests include machine learning, computational neuroscience, biomedical signal processing, and the brain-computer interface.

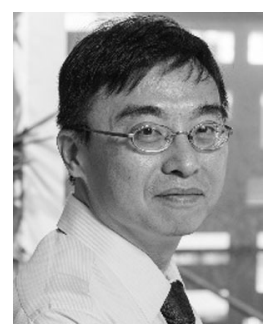

Dr. Chin-Teng Lin received the B.S. degree from National Chiao-Tung University (NCTU), Hsinchu, Taiwan, in 1986, and the Master and Ph.D. degrees in electrical engineering from Purdue University, West Lafayette, IN, USA, in 1989 and 1992, respectively. He is currently the Distinguished Professor of Faculty of Engineering and Information Technology, and the Co-Director of Center for Artificial Intelligence, University of Technology Sydney, Ultimo, NSW, Australia. He is also invited as Honorary Chair Professor of Electrical and Computer Engineering, NCTU, and Honorary Professorship of University of Nottingham. He has co-authored of Neural Fuzzy Systems (Prentice-Hall), and authored of Neural Fuzzy Control Systems with Structure and Parameter Learning (World Scientific), and more than 300 journal papers (Total Citation: 19,232, H-index: 64, i10-index: 243) in the areas of neural networks, fuzzy systems, brain computer interface, multimedia information processing, and cognitive neuro-engineering, including more than 120 IEEE journal papers. He was the recipient of the IEEE Fuzzy Systems Pioneer Awards in 2017. He was the Editor-in-Chief of the IEEE TRANSACTIONS ON FUZZY SYSTEMS from 2011 to 2016. He also served on the Board of Governors at the IEEE Circuits and Systems (CAS) Society in 2005-2008, the IEEE Systems, Man, Cybernetics (SMC) Society in 2003-2005, the IEEE Computational. Intelligence Society in 2008-2010, and the Chair of the IEEE Taipei Section in 2009-2010. He was the Distinguished Lecturer of the IEEE CAS Society from 2003 to 2005 and CIS Society from 2015-2017. He serves as the Chair of the IEEE CIS Distinguished Lecturer Program Committee in 2018. He served as the Deputy Editor-in-Chief of the IEEE Transactions on Circuits and Systems-II in 2006-2008. He was the Program Chair of the IEEE International Conference on Systems, Man, and Cybernetics in 2005 and General Chair of 2011 the IEEE International Conference on Fuzzy Systems. He was elevated to be an IEEE Fellow for his contributions to biologically inspired information systems in 2005, and was elevated International Fuzzy Systems Association Fellow in 2012. 